Supporting Information for

\title{
Different Single-Enzyme Conformational Dynamics upon Binding Hydrolyzable or Nonhydrolyzable Ligands
}

\author{
Sung Oh $\mathrm{Woo}^{1}$, Myungkeun $\mathrm{Oh}^{2}$, Lina Alhalhooly ${ }^{1}$, Jasmin Farmakes ${ }^{3}$, Arith J. Rajapakse ${ }^{4}$, \\ Zhongyu Yang ${ }^{3}$, Philip G. Collins ${ }^{4}$, Yongki Choi ${ }^{1,2, *}$ \\ ${ }^{1}$ Department of Physics, ${ }^{2}$ Materials and Nanotechnology Program, and ${ }^{3}$ Department of \\ Chemistry and Biochemistry, North Dakota State University, Fargo, North Dakota 58108, United \\ States \\ ${ }^{4}$ Department of Physics and Astronomy, University of California, Irvine, California 92696, \\ United States
}

\section{Materials and Methods}

Protein expression and purification. A pseudo wild-type T4 lysozyme mutant (C54T/C97A/S90C) was synthesized via site-directed mutagenesis and expressed from Escherichia coli cells as previously described. ${ }^{1-3}$ The purity of the expressed mutant was confirmed with gelelectrophoresis. The secondary structure of the mutant was confirmed with Circular Dichroism. The mutant was also confirmed to be functionally active with the commercial activity kit (Micrococcus lysodeikticus, ATCC No. 4698, Sigma-Aldrich), showing similar catalytic efficiency as compared to the wild-type protein.

Lysozyme substrate. Peptidoglycan from Micrococcus luteus, chitin from Pandalus borealis, and indole-3-propinoic acid was purchased from Sigma-Aldrich (St. Louis, MO) and used without further purification as a substrate to assay lysozyme activity. Each substrate was suspended in PBS (pH 7.5) to a final concentration of $25 \mu \mathrm{g} / \mathrm{ml}$. Typical experiments flushed the apparatus with 2 $\mathrm{mL}$ of substrate supernatant solution to insure the presence of excess substrate in the vicinity of the lysozyme-SWNT device.

Single-molecule nanocircuit fabrication. SWNTs were grown by chemical vapor deposition (CVD), followed by optical lithography for patterning electrodes and electron beam lithography for the electrodes passivation described previously. ${ }^{4,5}$ Devices were functionalized using a bifunctional linker, pyrene-maleimide. The pyrene functionality adheres to the surface of graphene strongly via $\pi-\pi$ stacking. The maleimide group can form stable thioether bonds with the free thiol of a cysteine sidechain in the protein. A solution of N-(1-pyrenyl)maleimide (Sigma Aldrich) in ethanol $(1 \mathrm{mM})$ was prepared. Devices were soaked in the solution for 30 min without agitation, and then washed with $0.1 \%$ Tween-20 (Acros Organics) in ethanol and de-ionized water for $5 \mathrm{~min}$ to remove excess pyrene-maleimide. Next, a solution of lysozyme (5.4 $\mu \mathrm{M}$ in PBS) prepared as 
described above was used for bioconjugation. At room temperature, pyrene-maleimidefunctionalized devices were soaked in the lysozyme solution for $60 \mathrm{~min}$. Following bioconjugation, devices were rinsed with wash buffer $\left(5 \mathrm{mM} \mathrm{KCl}, 10 \mathrm{mM} \mathrm{Na} 2 \mathrm{HPO}_{4}, 0.05 \%\right.$ Tween-20, pH 7) to remove nonspecifically adsorbed lysozyme, stored in the buffer, and neither dried nor imaged until the completion of measurements.

Device measurements and data analysis. To perform high-bandwidth electrical measurements, lysozyme SWNT devices were inserted into a home-built microfluidic flow cell. A patterned PDMS gasket was aligned over the SWNT chip, placing a $100-\mu \mathrm{m}$ wide microfluidic channel directly over the exposed region of the SWNT FET. Clamping the gasket in place confined liquid to the desired channel and isolated from source and drain contact pads. The drain electrode was biased at $100 \mathrm{mV}$ and the back gate electrode was grounded. Source-drain current $I(t)$ was measured using a FEMTO DLPCA-200 low noise preamplifier operating at $10^{8} \mathrm{~V} / \mathrm{A}$ gain and with a $1.8 \mu$ s rise time. This signal was digitized at $600 \mathrm{kHz}$ and stored for later analysis. After confirming electrical connectivity of the SWNT FET device, PBS was flushed into the microfluidic channel. Fluid inlets and outlets contained a combination of Pt counter electrodes and Pt pseudo-references, both of which were controlled and monitored using National Instruments data acquisition hardware (PCI-6281) and LabVIEW. Once the PBS or other fluid was in direct contact with the exposed SWNT channel, the device could be electrostatically gated by varying the Pt counter electrode. During measurements, the potential between the SWNT source electrode and the Pt pseudo-reference electrode was typically held at -0.1 to $-0.4 \mathrm{~V}$, a modest potential that left the p-type SWNT channel in the same doping state it would normally have in air. In every experiment, $I(t)$ data were collected in PBS to confirm that the FET had a steady and featureless baseline current. Satisfactory devices exhibited DC resistances of 0.5 to $5.0 \mathrm{M} \Omega$ with no $I(t)$ features outside the 1/f noise spectrum that is normal for SWNTs. Data was collected for $600 \mathrm{~s}$ in PBS immediately before the introduction of substrates, and then for at least $600 \mathrm{~s}$ after flushing 2 $\mathrm{mL}$ of substrate solution through the fluid cell. At the conclusion of experiments, $I(t)$ was rechecked after flushing the substrate with $2 \mathrm{~mL}$ of PBS. No data was collected during fluid exchanges or flushing of the cell. In this operating limit of small, DC potentials, we assume that the SWNT FET is a passive transducer of lysozyme's activity. A previous study ${ }^{6}$ showed that the $I(t)$ signals studied here are generated by these two sidechains, K83 and R119, located close to the SWNT attachment but far from lysozyme's catalytically active site. In the presence of thermal fluctuations and ionic screening, we do not believe any consequential back-action propagates from a DC-biased SWNT, through these sidechains, and back to the active site. The analysis has been performed using a 10-Hz, digital highpass filter. After filtering, multi-peak fitting was performed on histograms of 1-second data sets to estimate the second-by-second average position of $I(t)$ for open, intermediate, and closed conformations. Combining the highpass filtering with this peakfinding routine enhanced the accurate identification of events in $I(t)$ without requiring adaptive or more aggressive filters that introduce artifacts.

AFM imaging. The devices were imaged using a commercial atomic force microscope (NT-MDT NTEGRA). Prior to the imaging, the devices were dried by flowing nitrogen gas for $5 \mathrm{~min}$. The semi-contact mode AFM measurements were performed under ambient conditions with AFM probes of resonant frequency $150-300 \mathrm{kHz}$ (Budget sensors). The images were flattened to eliminate the background noise and tilt from the surface using all unmasked portion of scan lines to calculate individual least-square fit polynomials for each line. 


\begin{tabular}{|c|c|c|c|c|c|c|}
\hline & \multicolumn{5}{|c|}{ Catalytic Process } & \multirow{2}{*}{ Closed } \\
\hline \multirow{2}{*}{ State } & \multirow{2}{*}{ Open } & \multicolumn{5}{|c|}{ Intermediate } \\
\cline { 3 - 6 } & & closing_fail & closing & opening & opening_fail & \\
\hline \hline mean $(\mathrm{ms})$ & 11.89 & 0.88 & 0.91 & 0.92 & 0.83 & 3.73 \\
\hline$\sigma(\mathrm{ms})$ & 14.12 & 0.74 & 0.81 & 1.01 & 0.92 & 4.18 \\
\hline
\end{tabular}

\begin{tabular}{|c|c|c|c|c|c|c|}
\hline & \multicolumn{5}{|c|}{ Nonproductive process } & \multirow{2}{*}{ Closed } \\
\hline \multirow{2}{*}{ State } & \multirow{2}{*}{ Open } & \multicolumn{5}{|c|}{ Intermediate } \\
\cline { 3 - 6 } & & closing_fail & closing & opening & opening_fail & \\
\hline \hline mean (ms) & 1.60 & 0.30 & 0.38 & 0.41 & 0.25 & 0.13 \\
\hline$\sigma(\mathrm{ms})$ & 2.10 & 0.73 & 0.76 & 1.21 & 0.39 & 0.18 \\
\hline
\end{tabular}

Table S1. Timing of lysozyme activity with the peptidoglycan substrate.

\begin{tabular}{|c|c|c|c|c|c|}
\hline \multirow{2}{*}{ Parameter } & \multicolumn{3}{|c|}{ chitin } & \multicolumn{2}{c|}{ IPA } \\
\cline { 2 - 6 } & Open (0) & 1st excited (1) & 2nd excited (2) & Open & excited \\
\hline mean (ms) & 1.73 & 0.16 & 0.09 & 1.22 & 0.12 \\
\hline$\sigma(\mathrm{ms})$ & 2.06 & 0.14 & 0.06 & 1.82 & 0.09 \\
\hline
\end{tabular}

Table S2. Timing of lysozyme's conformational activity with chitin and IPA.

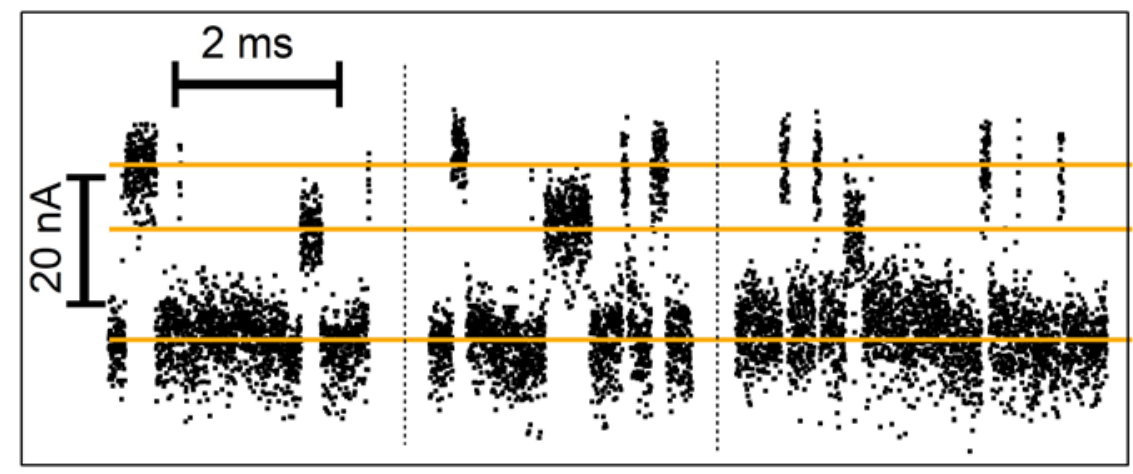

Figure S1. Example $\Delta I(t)$ signal for the interrupt pause events during the fast, nonproductive motions.

\section{References}

(1) Yang, Z.; Jiménez-Osés, G.; López, C. J.; Bridges, M. D.; Houk, K. N.; Hubbell, W. L., Long-Range Distance Measurements in Proteins at Physiological Temperatures Using Saturation Recovery EPR Spectroscopy. J. Am. Chem. Soc. 2014, 136, 15356-15365.

(2) Choi, Y.; Moody, I. S.; Sims, P. C.; Hunt, S. R.; Corso, B. L.; Seitz, D. E.; Blaszcazk, L. C.; Collins, P. G.; Weiss, G. A., Single-Molecule Dynamics of Lysozyme Processing Distinguishes Linear and Cross-Linked Peptidoglycan Substrates. J. Am. Chem. Soc. 2012, 134, 2032-2035.

(3) Woo, S. O.; Froberg, J.; Pan, Y.; Tani, S.; Goldsmith, B. R.; Yang, Z.; Choi, Y., Protein Detection Using Quadratic Fit Analysis near the Dirac Point of Graphene Field-Effect Biosensors. ACS Appl. Electron. Mater. 2020, 2, 913-919. 
(4) Goldsmith, B. R.; Coroneus, J. G.; Khalap, V. R.; Kane, A. A.; Weiss, G. A.; Collins, P. G., Conductance-controlled point functionalization of single-walled carbon nanotubes.

Science 2007, 315, 77-81.

(5) Choi, Y.; Moody, I. S.; Sims, P. C.; Hunt, S. R.; Corso, B. L.; Weiss, G. A.; Collins, P. G., Single-Molecule Lysozyme Dynamics Monitored by an Electronic Circuit. Science 2012, 335, 319-324

(6) Choi, Y.; Olsen, T. J.; Sims, P. C.; Moody, I. S.; Corso, B. L.; Dang, M. N.; Weiss, G. A.; Collins, P. G., Dissecting Single-Molecule Signal Transduction in Carbon Nanotube Circuits with Protein Engineering. Nano Lett. 2013, 13, 625-631. 